\title{
A!
}

This is an electronic reprint of the original article.

This reprint may differ from the original in pagination and typographic detail.

Murto, Pauli; Välimäki, Juuso

\section{Large all-pay auctions with IPV bidders}

Published in:

Journal of Economic Theory

DOI:

10.1016/j.jet.2017.03.008

Published: 01/05/2017

Document Version

Peer reviewed version

Published under the following license:

CC BY-NC-ND

Please cite the original version:

Murto, P., \& Välimäki, J. (2017). Large all-pay auctions with IPV bidders. Journal of Economic Theory, 169, 629-

640. https://doi.org/10.1016/j.jet.2017.03.008

This material is protected by copyright and other intellectual property rights, and duplication or sale of all or part of any of the repository collections is not permitted, except that material may be duplicated by you for your research use or educational purposes in electronic or print form. You must obtain permission for any other use. Electronic or print copies may not be offered, whether for sale or otherwise to anyone who is not an authorised user. 


\title{
Large All-Pay Auctions with IPV Bidders
}

\author{
Pauli Murto* Juuso Välimäki
}

March 7, 2017

\begin{abstract}
This note analyzes different types of all-pay auctions when the number of bidders becomes large. We compute the distributions of the highest bids for the first-price all pay auction and we show that the expected payment made by the winning bidder converges to half of the total payments. In the second-price all-pay auction (the war of attrition), the highest bid amounts to about 35.5 $\%$ of the total revenues. We also compute the payments for all-pay auctions with multiple prizes.
\end{abstract}

KEYWORDS: all-pay auction, large auctions

JEL Classification: D44

\section{Introduction}

All-pay auctions can be seen as the simplest type of a rent seeking contest. A number of bidders choose how much to bid in exchange for a favor with the understanding that the highest bidder is granted the favor. The advantage of this extremely simple model is that well-known results from auction theory can be used to characterize equilibrium bidding.

In this note, we compute the equilibrium outcomes for large all-pay auctions where a number of privately informed players make a bid for an object. All bidders

*Aalto University School of Business, pauli.murto@aalto.fi

$\dagger$ †alto University School of Business, juuso.valimaki@aalto.fi 
including the losing ones have to pay their own bid. Since almost all bidders are losers in a bidding contest with large numbers of participants, the bidders will have to be extremely careful in preparing their bids: only the types with values close to the top of the support of valuations make bids that differ significantly from zero.

Our base model has a single object and $N$ ex ante symmetric bidders with private valuations drawn independently from a fixed interval. We compute the limiting random outcome for the model as $N \rightarrow \infty$. In light of the revenue equivalence theorem, it is no surprise that in any symmetric equilibrium of the model, the expected revenue in such auctions converges to the highest valuation in the support of the bidders valuations. More interestingly, we give an explicit formula for the distribution of the $K$ highest bids for the limiting game where $N$ grows towards infinity. The expected revenue from the winning highest bid is half of the total revenue. More generally, the $k^{\text {th }}$ highest bid brings in expectation $\frac{1}{2^{k}}$ of the total revenue.

We get this revenue result by combining the well known formula of the symmetric bidding equilibrium for all-pay auctions with the asymptotic characterization of the distribution of the perceived winning probabilities within the population of players. We normalize the value of the object to lie in the unit interval, and as a result, the highest bidders assign a value close to unity to the object. In an all-pay auction, a bidder breaks even if she bids her value of the object multiplied by the probability of winning. In large auctions, bidders cannot make a positive rent. As a result, the winning bidder must make a bid equal to her probability of winning. We show that in large auctions, the interim winning probability of the bidder with the highest order statistic converges in distribution to the uniform distribution. In other words, the perceived winning probability of the winner in the all-pay auction is uniformly distributed. We also show that conditional on the $k^{\text {th }}$ highest bidder perceiving a probability $x$ of winning, the $(k+1)^{s t}$ highest bidder's perceived probability of winning is uniform on $[0, x]$.

There is a large literature on standard auctions with many bidders and most of the economic questions in the IPV setting are settled. ${ }^{1}$ The analysis of all-pay auctions is more complicated than the analysis of standard auctions where only the winner pays. In standard auctions, the bidding functions have a well defined continuous

\footnotetext{
${ }^{1}$ Our private values setup is an easy special case of Bali \& Jackson (2002).
} 
limit. In the second-price auction, the bid equals own valuation regardless of the number of bidders. In first-price auctions, bids also converge to own valuation as the number of players grows large. In all-pay auctions, this is not true. The limit of the equilibrium bidding functions is discontinuous. At all fixed types strictly below the value of the object, the symmetric equilibrium bid converges to zero. The bid of the highest type converges to the value of the object. A meaningful analysis of the auction then requires us to focus on the bids of the bidders at an appropriate distance (that depends on the number of bidders) from the top of the distribution.

In a recent paper, Olszewski \& Siegel (2016) study contests with large numbers of bidders. In that paper, the number of prizes also increases with the number of bidders. As a result, the outcome for an individual bidder depends on the fraction of bidders submitting a higher bid. This implies that the bid distribution that each individual bidder is facing is deduced by using the law of large numbers. With a single prize, the relevant statistic for an individual bidder is the highest order statistic in the bids by others. As a result, the method of analysis in our paper is quite different from theirs. ${ }^{2}$

We conclude the note by deriving revenue results for the second-price all-pay auction (also known as the silent war of attrition) and for an auction with $n$ possibly different prizes. ${ }^{3}$ Similar steps as in the case of all-pay auction allow us to characterize the expected payments by the $K$ highest bidders. In the war of attrition, the limiting density of the highest bid is the exponential distribution, and the winning bidder's expected payment is $35.5 \%$ of the value of the object. In the multi-prize auction, we derive a simple formula for the expected values of the highest bids in the general case.

\footnotetext{
${ }^{2} \mathrm{An}$ analogy can be drawn to large affiliated $k+1^{\text {st }}$ price auctions where the highest bid remains random in a single-object auction, but converges to the true value as the number of objects sold increases (see Pesendorfer \& Swinkels (1997)).

${ }^{3}$ We thank Hannu Vartiainen for suggesting that we analyze the war of attrition and the Associate Editor for the suggestion of covering the contest with $n$ prizes.
} 


\section{Model}

There are $N$ ex ante identical bidders with private valuations $\theta_{i}$ drawn independently from a common distribution $F(\cdot)$ with support $[0,1]$, and a continuous density $f(\cdot)$. We denote by $\theta_{N}^{(k)}$ the $k^{\text {th }}$ order statistic amongst $\left(\theta_{1}, \ldots, \theta_{N}\right)$.

Each bidder $i$ submits a bid $b_{i}$ and the payoff to bidder $i$ of type $\theta_{i}$ given a vector of bids $b=\left(b_{1}, \ldots, b_{N}\right)$ is given by

$$
u_{i}\left(\theta_{i}, b\right)=\left\{\begin{array}{l}
\theta_{i}-b_{i} \text { if } b_{i}>b_{j} \text { for all } j \neq i \\
\frac{1}{n} \theta_{i}-b_{i} \text { if } b_{i} \text { ties for highest bid with }(n-1) \text { other bids } \\
-b_{i} \text { otherwise. }
\end{array}\right.
$$

We look for a symmetric Bayesian Nash Equilibrium where all bidders use the same equilibrium strategy $b_{i}\left(\theta_{i}\right)=b\left(\theta_{i}\right)$.

We are interested in characterizing for each fixed $K$ the distribution of the $K$ highest bids along a sequence of games where $N \rightarrow \infty$. Let $b_{N}^{(k)}$ stand for the $k^{\text {th }}$ order statistic amongst $\left(b_{N}\left(\theta_{1}\right), \ldots, b_{N}\left(\theta_{N}\right)\right)$, where $b_{N}(\cdot)$ is a symmetric equilibrium strategy in the game with $N$ players. Let

$$
b^{(k)}=\lim _{N \rightarrow \infty} b_{N}^{(k)},
$$

where the limit is in the sense of convergence in distribution. We call the vector of random variables $\left(b^{(1)}, \ldots, b^{(K)}\right)$ the limiting equilibrium outcome of the game. Note that we are not defining equilibrium strategies in the limit game, but characterizing the limit of equilibrium bids along the sequence of finite games as $N$ grows large.

\section{Distribution of perceived winning probabilities}

Before analyzing the equilibrium bids, we explain the key step in our analysis. Instead of working with the order statistics of signals, we work with the order statistics of perceived winning probabilities. Given strictly increasing bidding strategies, the probability of winning is the probability that all the other players have lower signals. Let $\alpha_{N}(\theta)$ denote the winning probability of a player with signal $\theta$ :

$$
\alpha_{N}(\theta):=F(\theta)^{N-1}
$$


and let $\alpha_{N}^{(k)}$ denote the $k^{\text {th }}$ highest subjective winning probability:

$$
\alpha_{N}^{(k)}:=\alpha_{N}\left(\theta_{N}^{(k)}\right)=F\left(\theta_{N}^{(k)}\right)^{N-1}
$$

Since the type distribution $F$ has support [0,1], the $k^{\text {th }}$ order statistic $\theta_{N}^{(k)}$ from any sample of size $N$ with $k<N$ is a random variable on $[0,1]$, and consequently so is $\alpha_{N}^{(k)}=F\left(\theta_{N}^{(k)}\right)^{N-1}$. For all fixed $k, \theta_{N}^{(k)}$ converges to the degenerate distribution on 1 as $N \rightarrow \infty$. Similarly, for all $\theta \in(0,1), F(\theta)^{N-1} \rightarrow 0$ as $N \rightarrow \infty$. We now show that the composite random variable $\alpha_{N}^{(k)}$ has a non-degenerate limiting distribution on $[0,1]$ as $N \rightarrow \infty$.

Proposition 1 characterizes the limiting joint distribution for $\left(\alpha_{N}^{(1)}, \ldots, \alpha_{N}^{(K)}\right)$ on $[0,1]^{K}$ as $N \rightarrow \infty$. We let $U(a, b)$ denote the uniform distribution on $[a, b]$, and the convergence denoted by $\stackrel{d}{\rightarrow}$ is understood to be in the sense of convergence in distribution.

Proposition 1 For any i.i.d. sample $\left(\theta_{1}, \ldots, \theta_{N}\right)$ from a fixed distribution $F(\cdot)$,

$$
\alpha_{N}^{(k)} \stackrel{d}{\rightarrow} \alpha^{(k)} \text { as } N \rightarrow \infty
$$

where $\alpha^{(0)}=1$ and $a^{(k)}, k=1, \ldots, K$ is a random variable with conditional distribution

$$
\left(\alpha^{(k)} \mid \alpha^{(k-1)}\right) \sim U\left(0, \alpha^{(k-1)}\right) .
$$

Proof. By the definition of $\alpha_{N}^{(1)}$, we have:

$$
\begin{aligned}
\operatorname{Pr}\left(\alpha_{N}^{(1)} \leq x\right) & =\operatorname{Pr}\left[F\left(\theta_{N}^{(1)}\right)^{N-1} \leq x\right] \\
& =\operatorname{Pr}\left[F\left(\theta_{N}^{(1)} \leq x^{\frac{1}{N-1}}\right] .\right.
\end{aligned}
$$

Since the event $F\left(\theta_{N}^{(1)}\right) \leq x^{\frac{1}{N-1}}$ is the event that $F\left(\theta_{i}\right) \leq x^{\frac{1}{N-1}}$ for all $i=1, \ldots, N$ and since the signals are independently distributed, we have

$$
\operatorname{Pr}\left[F\left(\theta_{N}^{(1)}\right) \leq x^{\frac{1}{N-1}}\right]=\left(\operatorname{Pr}\left[F(\theta) \leq x^{\frac{1}{N-1}}\right]\right)^{N}=\left(x^{\frac{1}{N-1}}\right)^{N}=x^{\frac{N}{N-1}}
$$


where the second equality follows from the fact that if $Y$ is a random variable with c.d.f. $F(\cdot)$, then $F(Y)$ is distributed uniformly on $[0,1]$. Therefore, for any $x \in[0,1]$, we have

$$
\operatorname{Pr}\left(\alpha_{N}^{(1)} \leq x\right)=x^{\frac{N}{N-1}}
$$

Taking limits as $N \rightarrow \infty$ gives

$$
\lim _{N \rightarrow \infty} \operatorname{Pr}\left(\alpha_{N}^{(1)} \leq x\right)=\lim _{N \rightarrow \infty} x^{\frac{N}{N-1}}=x,
$$

and therefore

$$
\alpha_{N}^{(1)} \stackrel{d}{\rightarrow} \alpha^{(1)} \sim U(0,1)
$$

Consider next the distribution of $\alpha_{N}^{(k)}$ conditional on $\alpha_{N}^{(k-1)}$. Fix $x$ and $y$ with $0<x<y<1$. Then we have

$$
\begin{aligned}
\operatorname{Pr}\left[\alpha_{N}^{(k)} \leq x \mid \alpha_{N}^{(k-1)}=y\right] & =\operatorname{Pr}\left[F\left(\theta_{N}^{(k)}\right)^{N-1} \leq x \mid F\left(\theta_{N}^{(k-1)}\right)^{N-1}=y\right] \\
& =\operatorname{Pr}\left[F\left(\theta_{N}^{(k)}\right) \leq x^{\frac{1}{N-1}} \mid F\left(\theta_{N}^{(k-1)}\right)=y^{\frac{1}{N-1}}\right] \\
& =\operatorname{Pr}\left[\theta_{N}^{(k)} \leq F^{-1}\left(x^{\frac{1}{N-1}}\right) \mid \theta_{N}^{(k-1)}=F^{-1}\left(y^{\frac{1}{N-1}}\right)\right]
\end{aligned}
$$

Since we have assumed that $F$ is atomless and has full support [0,1], its inverse function $F^{-1}$ is well defined.

Note that if $\theta_{N}^{(k-1)}=F^{-1}\left(y^{\frac{1}{N-1}}\right)$, then there are exactly $(N-(k-1))$ signals with a value at or below $F^{-1}\left(y^{\frac{1}{N-1}}\right)$. The event $\theta_{N}^{(k)} \leq F^{-1}\left(x^{\frac{1}{N-1}}\right)<\theta_{N}^{(k-1)}$ is the event that each of those $(N-(k-1))$ lowest signals is further down below $F^{-1}\left(x^{\frac{1}{N-1}}\right)$. Since individual signals are independently distributed random variables, we then have

$$
\begin{aligned}
& \operatorname{Pr}\left[\theta_{N}^{(k)} \leq F^{-1}\left(x^{\frac{1}{N-1}}\right) \mid \theta_{N}^{(k-1)}=F^{-1}\left(y^{\frac{1}{N-1}}\right)\right] \\
= & \left(\operatorname{Pr}\left[\theta \leq F^{-1}\left(x^{\frac{1}{N-1}}\right) \mid \theta \leq F^{-1}\left(y^{\frac{1}{N-1}}\right)\right]\right)^{N-(k-1)} .
\end{aligned}
$$

Finally, since the event $\left\{\theta \leq F^{-1}\left(x^{\frac{1}{N-1}}\right)\right\}$ is a subset of the event $\left\{\theta \leq F^{-1}\left(y^{\frac{1}{N-1}}\right)\right\}$, 
Bayes' rule gives:

$$
\begin{aligned}
& \operatorname{Pr}\left[\theta \leq F^{-1}\left(x^{\frac{1}{N-1}}\right) \mid \theta \leq F^{-1}\left(y^{\frac{1}{N-1}}\right)\right] \\
= & \frac{\operatorname{Pr}\left[\theta \leq F^{-1}\left(x^{\frac{1}{N-1}}\right)\right]}{\operatorname{Pr}\left[\theta \leq F^{-1}\left(y^{\frac{1}{N-1}}\right)\right]}=\frac{\operatorname{Pr}\left[F(\theta) \leq x^{\frac{1}{N-1}}\right]}{\operatorname{Pr}\left[F(\theta) \leq y^{\frac{1}{N-1}}\right]} \\
= & \left(\frac{x}{y}\right)^{\frac{1}{N-1}},
\end{aligned}
$$

where the last equality follows again from the fact that if $Y$ has c.d.f. $F(\cdot)$, then $F(Y)$ is a uniform r.v. on $[0,1]$.

Combining (1) - (3), we have

$$
\operatorname{Pr}\left[\alpha_{N}^{(k)} \leq x \mid \alpha_{N}^{(k-1)}=y\right]=\left[\left(\frac{x}{y}\right)^{\frac{1}{N-1}}\right]^{N-(k-1)}=\left(\frac{x}{y}\right)^{\frac{N-(k-1)}{N-1}} .
$$

Hence:

$$
\lim _{N \rightarrow \infty} \operatorname{Pr}\left[\alpha_{N}^{(k)} \leq x \mid \alpha_{N}^{(k-1)}=y\right]=\lim _{N \rightarrow \infty}\left(\frac{x}{y}\right)^{\frac{N-(k-1)}{N-1}}=\frac{x}{y}
$$

and so $\alpha_{N}^{(k)}, k=2,3, \ldots$ converge in distribution to random variables $\alpha^{(k)}$ for which

$$
\left(\alpha^{(k)} \mid \alpha^{(k-1)}\right) \sim U\left(0, \alpha^{(k-1)}\right) .
$$

Proposition 1 characterizes the random variables $\alpha^{(k)}$ recursively in terms of their conditional distributions. To compute the bid distributions for different types of allpay auctions, we will also need the unconditional distributions of $\alpha^{(k)}$. It is convenient to express these unconditional distributions in terms of a logaritmic transformation:

Corollary 1 The random variable $-\ln \left(\alpha^{(k)}\right)$ is distributed according to a Gamma distribution with parameters $(k, 1)$.

Proof. We can write

$$
\begin{aligned}
\operatorname{Pr}\left[-\ln \left(\alpha^{(k)}\right) \leq x\right] & =\operatorname{Pr}\left[-\ln \left(\frac{\alpha^{(k)}}{\alpha^{(k-1)}} \cdot \ldots \cdot \frac{\alpha^{(2)}}{\alpha^{(1)}} \cdot \alpha^{(1)}\right) \leq x\right] \\
& =\operatorname{Pr}\left(-\ln \frac{\alpha^{(k)}}{\alpha^{(k-1)}}-\ldots-\ln \frac{\alpha^{(2)}}{\alpha^{(1)}}-\ln \alpha^{(1)} \leq x\right) .
\end{aligned}
$$


It follows from Proposition 1 that $\frac{\alpha^{(k)}}{\alpha^{(k-1)}}, \ldots, \alpha^{(1)}$ are $k$ independent uniformly distributed random variables. Hence, $-\ln \left(\alpha^{(k)}\right)$ is a sum of $k$ independent exponentially distributed random variables, i.e. Gamma with parameters $(k, 1)$.

\section{All-Pay Auction}

We now use the result of the previous section to analyze the bid distribution of a large all-pay auction. By a standard incentive compatibility argument, the symmetric equilibrium bid distribution $b_{N}(\cdot)$ must be an increasing function for all $N$. By a simple undercutting argument, the bid distribution cannot have ties. Hence we know that $b_{N}\left(\theta_{i}\right)$ must be strictly increasing, and as a result, the outcome in the auction is efficient. In any symmetric strictly monotonic equilibrium, the lowest bidder $\theta=0$ earns a zero expected payoff. This allows us to conclude immediately that the limiting expected revenue in the game is 1 . The easiest way to see this is by using the revenue equivalence theorem and the obvious observation that the expected payment in the second price auction converges to 1 as the second highest order statistic converges to the upper boundary of the support.

It is more interesting to characterize the limiting bid functions. Utilizing the revenue equivalence principle, we can write the payoff of type $\theta_{i}$ in a symmetric equilibrium as follows (see e.g. Krishna (2002) or Milgrom (2004)):

$$
\theta_{i}\left(F\left(\theta_{i}\right)\right)^{N-1}-b_{N}\left(\theta_{i}\right)=\int_{0}^{\theta_{i}}(F(s))^{N-1} d s .
$$

Hence the symmetric equilibrium bid is

$$
b_{N}\left(\theta_{i}\right)=\theta_{i}\left(F\left(\theta_{i}\right)\right)^{N-1}-\int_{0}^{\theta_{i}} F(s)^{N-1} d s .
$$

Observe that as $N$ grows, the term on the right hand side capturing the information rent accruing to the bidder of type $\theta_{i}$ vanishes, and the only remaining task is to evaluate $\theta_{i}\left(F\left(\theta_{i}\right)\right)^{N-1}$ for large $N .^{4}$

The important term in the bid function is $\left(F\left(\theta_{i}\right)\right)^{N-1}$, which we have denoted by $\alpha_{N}\left(\theta_{i}\right)$. This is simply the probability that all the other players have a signal lower

$$
{ }^{4} \int_{0}^{1}(F(s))^{N-1} d s \rightarrow 0 \text { since }(F(s))^{N-1} \rightarrow 0 \text { a.e. }
$$


than $\theta_{i}$. The key point to notice here is that even though $\left(F\left(\theta_{i}\right)\right)^{N-1}$ vanishes for all $\theta_{i}<1$ as $N$ grows, the largest realized types also converge to 1 as $N$ grows and an argument evaluating the bid at the highest order statistic is needed. As we have already shown above, $F\left(\theta_{N}^{(1)}\right)^{N-1}$ converges in distribution to $\alpha^{(1)} \sim U(0,1)$, and hence also the highest bid converges to uniform:

$$
b^{(1)} \sim U(0,1) .
$$

By the same argument, $F\left(\theta_{N}^{(k)}\right)^{N-1}$ converges to $\alpha^{(k)}$, and hence

$$
b_{N}\left(\theta_{N}^{(k)}\right) \rightarrow F\left(\theta_{N}^{(k)}\right)^{N-1} \stackrel{d}{\longrightarrow} \alpha^{(k)} .
$$

This means that for $k=2,3, \ldots$,

$$
\left(b^{(k)} \mid b^{(k-1)}\right) \sim U\left(0, b^{(k-1)}\right) .
$$

From these expressions we can also easily compute recursively the unconditional expectation of $b^{(k)}$ :

$$
\mathbb{E}\left(b^{(k)}\right)=\mathbb{E}_{b^{(k-1)}}\left(\mathbb{E} b^{(k)} \mid b^{(k-1)}\right)=\mathbb{E}_{b^{(k-1)}} \frac{b^{(k-1)}}{2}=\frac{1}{2} \mathbb{E}\left(b^{(k-1)}\right) .
$$

Since $\mathbb{E}\left(b^{(1)}\right)=\frac{1}{2}$, we get

$$
\mathbb{E}\left(b^{(k)}\right)=\frac{1}{2^{k}} \text { for } k=1,2, \ldots
$$

Collecting the results, we have proved the following proposition.

Proposition 2 In the symmetric equilibrium of an all-pay auction with $N$ bidders with independent private values drawn from the same distribution $F(\cdot)$ on $[0,1]$, the winning bid $b_{N}^{(1)}$ converges in distribution to a uniform random variable on $[0,1]$ and the conditional distribution of the $k^{\text {th }}$ highest bid $b_{N}^{(k)}$ given $\left(b_{N}^{(1)}, \ldots, b_{N}^{(k-1)}\right)$ converges to a uniform distribution on $\left[0, b^{(k-1)}\right]$.

Note that since

$$
\sum_{k=1}^{\infty} \frac{1}{2^{k}}=1,
$$

this confirms that the expected total revenue is one, which we already know from the revenue equivalence theorem. 


\section{Extensions}

\subsection{Second-Price All-Pay Auction}

In this section, we keep the statistical model identical to the one in the previous section and change the payoffs to second-price all-pay auction also known as the silent war of attrition. This model captures a game where the bidders decide at each point whether to add an increment to their bids. The only information they have when deciding whether to remain in the game is whether all other bidders have dropped out of the game and stopped bidding or not. The last remaining bidder wins the game and pays the bid of the last losing bidder. ${ }^{5}$ The silent war of attrition has a symmetric equilibrium in strictly increasing strategies so we write the payoff directly under the assumption that bids are not tied. ${ }^{6}$

Let $b_{N}\left(\theta_{i}\right)$ be the symmetric equilibrium bidding strategy in the game with $N$ players. The equilibrium expected payoff to a player with type $\theta_{i}$ is then

$$
U\left(\theta_{i}\right)=\left(\theta_{i}-\mathbb{E}\left[b_{N}\left(\theta_{N}^{(2)}\right) \mid \theta_{i}=\theta_{N}^{(1)}\right]\right) \operatorname{Pr}\left(\theta_{i}=\theta_{N}^{(1)}\right)-b_{N}\left(\theta_{i}\right)\left(1-\operatorname{Pr}\left(\theta_{i}=\theta_{N}^{(1)}\right)\right) .
$$

To use the results of Section 3, we want to express the equilibrium bid in the limit $N \rightarrow \infty$ as a function of the perceived winning probability. This was easy in the all-pay auction, since there bids were equal to perceived winning probabilities. In the war of attrition, this is more involved since the payment of the winner is determined by the next highest bid, i.e. by the bidder with the highest perceived probability of winning amongst the other bidders. Let $b(\alpha)$ denote the bid of a player who perceives her winning probability to be $\alpha$. Denoting the second-highest perceived winning probability by $\alpha^{\prime}$, we note that the distribution of $\alpha^{\prime}$ conditional on $\alpha^{\prime}<\alpha$ is uniform on $[0, \alpha]$. Finally, noting that a bidder with perceived winning probability $\alpha$ wins with probability $\alpha$ and loses with the complementary probability $1-\alpha$ (in which case she pays her own bid $b(\alpha)$ ), we can solve for $b(\alpha)$ by equating the gains

\footnotetext{
${ }^{5}$ It is well known that with more than two bidders, the regular (i.e. "non-silent") war of attrition does not have a symmetric equilibrium. See Bulow \& Klemperer (1999) for details.

${ }^{6}$ Theorem 1 in Krishna \& Morgan (1997) shows this for a larger class of games that includes our IPV model.
} 
when winning to losses when not winning:

$$
\alpha\left(1-\mathbb{E}\left[b\left(\alpha^{\prime}\right) \mid \alpha^{\prime}<\alpha\right]\right)=(1-\alpha) b(\alpha)
$$

or

$$
\alpha\left(1-\int_{0}^{\alpha} \frac{b\left(\alpha^{\prime}\right)}{\alpha} d \alpha^{\prime}\right)-(1-\alpha) b(\alpha)=0 \text { for all } \alpha .
$$

Differentiating this with respect to $\alpha$ gives:

$$
b^{\prime}(\alpha)=\frac{1}{1-\alpha},
$$

which together with initial condition $b(0)=0$ gives:

$$
b(\alpha)=-\ln (1-\alpha) .
$$

By inverting this relationship we get

$$
\alpha=1-e^{-b(\alpha)} .
$$

Since $\alpha^{(1)} \sim U(0,1)$, we note that

$$
1-e^{-b\left(\alpha^{(1)}\right)} \sim U(0,1)
$$

which means that in the limit, the highest bid is distributed according to the exponential distribution. This is not unexpected since indifference between continuing and quitting in a war of attrition requires that the last remaining other player quits with a constant hazard rate. In our silent war of attrition model this translates into the requirement that the highest bid by the other bidders has a constant hazard rate.

Using the previous results on $\alpha^{(k)}$, we get the expectation of the $k^{\text {th }}$ highest bid by evaluating

$$
\mathbb{E} b^{(k)}=\mathbb{E}_{\alpha^{(k)}}\left[-\ln \left(1-\alpha^{(k)}\right)\right]
$$

Since

$$
-\ln (1-\alpha)=-\ln \left(1-e^{\ln \alpha}\right)=-\ln \left(1-e^{-(-\ln \alpha)}\right),
$$

and

$$
-\ln \left(\alpha^{(k)}\right) \sim \operatorname{Gamma}(k, 1)
$$


we can derive an expression for $\mathbb{E} b^{(k)}$ in an explicit form:

$$
\mathbb{E} b^{(k)}=\int_{0}^{\infty}-\frac{x^{k-1}}{k-1 !} e^{-x} \ln \left(1-e^{-x}\right) d x .
$$

Again, we summarize these results in the following proposition.

Proposition 3 In the symmetric equilibrium of a second-price all-pay auction and $N$ bidders with independent private types drawn from the same distribution $F(\cdot)$ on $[0,1]$, the expectation of the $k^{\text {th }}$ highest bid converges to

$$
\mathbb{E} b^{(k)}=\int_{0}^{\infty}-\frac{x^{k-1}}{k-1 !} e^{-x} \ln \left(1-e^{-x}\right) d x
$$

as $N \rightarrow \infty$.

We can compute the expectation of the second highest bid:

$$
\mathbb{E} b^{(2)}=2-\frac{\pi^{2}}{6} \approx .355
$$

This shows that the winner makes a considerably lower expected payment in the second price all-pay auction than in the first-price version of the game. The highest loser pays the same amount as the winner, and in expectation, this exceeds the payment in the first-price all-pay auction.

By comparing the payments across the two types of all-pay auctions, we see that the rates of convergence towards zero are quite similar. The tenth highest bidder pays roughly $.1 \%$ of the value in both auctions.

\subsection{All-Pay Auction with Multiple Prizes}

In this subsection we analyze the model, where each of the $n$ highest bidders receives a prize. ${ }^{7}$ Let the values of the prizes be $q_{1} \theta \geq q_{2} \theta \geq \ldots \geq q_{n} \theta$ for a bidder of type $\theta$. We say that this all-pay auction has prize structure $q=\left(q_{1}, \ldots, q_{n}\right)$. In the limit, the highest bidders have all valuations arbitrarily close to 1 and as a consequence,

\footnotetext{
${ }^{7}$ If we let the number of prizes vary with $N$ with $\frac{n(N)}{N} \rightarrow x>0$ as $N \rightarrow \infty$, the model becomes a variant of the large contest models in Olszewski \& Siegel (2016).
} 
the auction is competitive for all fixed $n$ in the sense that the expected rent for each individual bidder vanishes as $N \rightarrow \infty$. Therefore, we must have in the symmetric equilibrium:

$$
\sum_{m=0}^{n-1} q_{m+1} \operatorname{Pr}\left\{b^{(m+1)} \leq b \leq b^{(m)}\right\}=b \text { for all } b \in[0,1]
$$

where $b^{(0)}=1$. As in the previous sections, we want to characterize the joint distribution of $\left(b^{(1)}, \ldots, b^{(K)}\right)$ for some $K$.

In order to specify this distribution, we start with a statistical result about the number of bidders who believe their probability of having the highest signal exceeds $\alpha \in(0,1)$. We denote this random number of high bidders by $X(\alpha, N)$ and show that it converges to a Poisson distributed random variable as $N \rightarrow \infty$ :

Lemma 1 Fix $\alpha \in(0,1)$ and let

$$
\begin{aligned}
\theta^{*}(\alpha, N): & =\left\{\theta: F(\theta)^{N-1}=\alpha\right\} \text { and } \\
X(\alpha, N): & =\#\left\{i: \theta_{i}>\theta^{*}(\alpha, N)\right\} .
\end{aligned}
$$

Then, as $N \rightarrow \infty$,

$$
X(\alpha, N) \stackrel{d}{\rightarrow} X_{\alpha} \sim \text { Poisson }(-\ln (\alpha))
$$

Proof. By definition of $\theta^{*}(\alpha, N)$,

$$
F\left(\theta^{*}(\alpha, N)\right)^{N-1}=\alpha,
$$

and hence

$$
F\left(\theta^{*}(\alpha, N)\right)=\alpha^{\frac{1}{N-1}}
$$

We have therefore

$$
\operatorname{Pr}\left(\theta_{i}>\theta^{*}(\alpha, N)\right)=1-F\left(\theta^{*}(\alpha, N)\right)=1-\alpha^{\frac{1}{N-1}}
$$

and hence $X(\alpha, N)$ is distributed according to

$$
X(\alpha, N) \sim \operatorname{Bin}\left(N, 1-\alpha^{\frac{1}{N-1}}\right) .
$$


Since

$$
N \cdot\left(1-\alpha^{\frac{1}{N-1}}\right) \underset{N \rightarrow \infty}{\rightarrow}-\ln (\alpha)
$$

the result follows from the fact that $\operatorname{Bin}(n, p)$ converges to Poisson $(\theta)$ when $n \rightarrow \infty$, $n p \rightarrow \theta$.

The interpretation of the result is the following. If $\alpha$ is a player's perceived probability of having the highest signal in a large auction, then the number of other players who have an even higher signal is distributed according to a Poisson distribution with parameter $-\ln (\alpha):=\varphi(\alpha)$. This allows us to calculate the expected winnings $\Pi(\alpha)$ in the auction for a bidder with perceived probability of winning $\alpha$ :

$$
\Pi(\alpha)=\sum_{m=0}^{n-1} q_{m+1} \operatorname{Pr}\left(X_{\alpha}=m\right)=\sum_{m=0}^{n-1} q_{m+1} \frac{\varphi(\alpha)^{m} e^{-\varphi(\alpha)}}{m !} .
$$

Since the auction is competitive, each bidder bids her expected winnings, i.e. $b(\alpha)=$ $\Pi(\alpha)$. From Corollary 1, we know that

$$
\varphi^{(k)}:=\varphi\left(\alpha^{(k)}\right) \sim \operatorname{Gamma}(k, 1),
$$

which gives us the formula for expected payment for the $k^{\text {th }}$ highest bidder:

$$
\begin{aligned}
\mathbb{E} b^{(k)} & =\mathbb{E} \Pi\left(\alpha^{(k)}\right)=\int_{0}^{\infty} \frac{x^{k-1}}{(k-1) !} e^{-x}\left(\sum_{m=0}^{n-1} q_{m+1} \frac{x^{m} e^{-x}}{m !}\right) d x \\
& =\sum_{m=0}^{n-1}\left(\begin{array}{c}
k+m-1 \\
m
\end{array}\right) \frac{q_{m+1}}{2^{k+m}} .
\end{aligned}
$$

Again, we can summarize:

Proposition 4 In the symmetric equilibrium of an all-pay auction with prize structure $q=\left(q_{1}, \ldots, q_{n}\right)$ and $N$ bidders with independent private types drawn from the same distribution $F(\cdot)$ on $[0,1]$, the expectation of the $k^{\text {th }}$ highest bid converges to

$$
\mathbb{E} b^{(k)}=\sum_{m=0}^{n-1}\left(\begin{array}{c}
k+m-1 \\
m
\end{array}\right) \frac{q_{m+1}}{2^{k+m}} .
$$

as $N \rightarrow \infty$.

As an illustration, Figure 1 shows the expected values of 30 highest bids in a contest with $n$ equally valued prizes with several different values of $n$. 


\section{References}

Bali, Valentina \& Matthew Jackson. 2002. "Asymptotic Revenue Equivalence in Auctions." Journal of Economic Theory 106(1):161 - 176.

Bulow, Jeremy \& Paul Klemperer. 1999. "The Generalized War of Attrition." American Economic Review 89(1):175-189.

Krishna, V. 2002. Auction Theory. Academic Press.

Krishna, Vijay \& John Morgan. 1997. "An Analysis of the War of Attrition and the All-Pay Auction." Journal of Economic Theory 72(2):343 - 362.

Milgrom, P. 2004. Putting Auction Theory to Work. Cambridge University Press.

Olszewski, Wojciech \& Ron Siegel. 2016. "Large Contests." Econometrica 84(2):835854.

Pesendorfer, W. \& J. Swinkels. 1997. "The Loser's Curse and Information Aggregation in Common Value Auctions." Econometrica 65(6):1247-1282. 


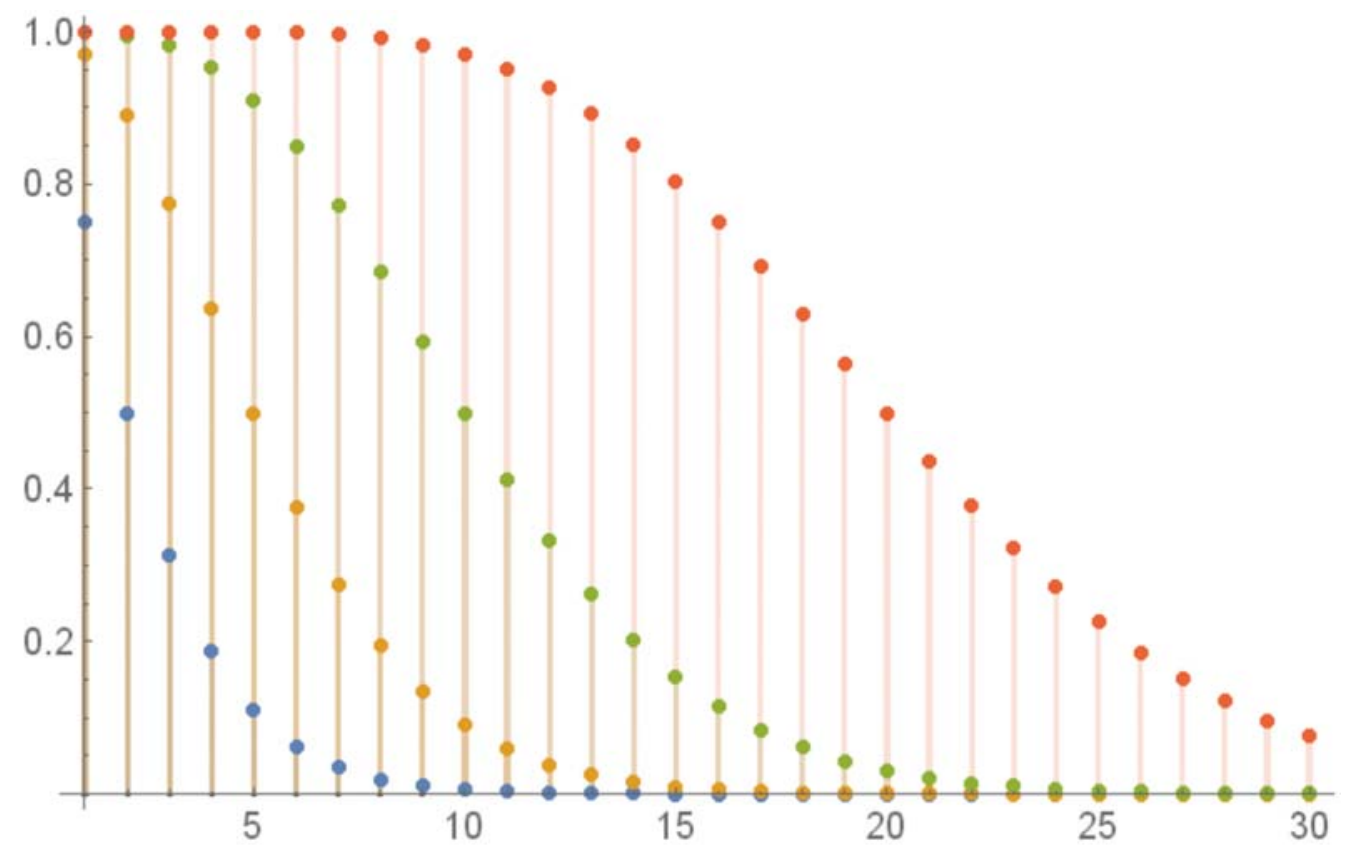

Figure 1: Expected highest bids $\mathbb{E} b^{(k)}, k=1, \ldots, 30$, for an all-pay auction with $n$ equally valuable prizes. Here $n$ takes values $n=2$ (blue), $n=5$ (yellow), $n=10$ (green), and $n=20$ (red). 